\title{
Determination of cable tensile forces using paraseismic effects
}

\author{
Daniel Papán ${ }^{1 *}$, Zuzana Papánová2 \\ ${ }^{1}$ University of Žilina, Faculty of Civil Engineering, Department of Structural Mechanics and Applied \\ Mathematics, Univerzitná 8215/1, 01026 Žilina Slovakia \\ ${ }^{2}$ University of Žilina, Faculty of Civil Engineering, Department of Structural Mechanics and Applied \\ Mathematics, Univerzitná 8215/1, 01026 Žilina Slovakia
}

\begin{abstract}
By construction or by reconstruction of civil structures can be paraseismic load generated. This effect may causes cracks in buildings standing near the source of the vibrations. Numerical simulation is one of the possibilities how can be this problem analyzed. The vibrations velocities of source transmitted to the surrounding buildings can be used as excitation to the numerical simulations. In the paper is presented the case study of the assessment of the tension forces in load bearing cables in real civil structure. This estimation is based on theoretical and experimental dynamic equations comparison.
\end{abstract}

\section{Introduction}

The case study was realized in one of the steel halls for Jaguar Land Rover Company in Nitra. The purpose of the research was the axial tensile forces in steel suspension cables determined. This steel suspension cables system supports the self-supporting steel grate construction over the production of car seats parts in the industrial operation line. Dynamic diagnosis the whole system is required because lifting devices and subsequent tensometric measurements cannot be used. [1]
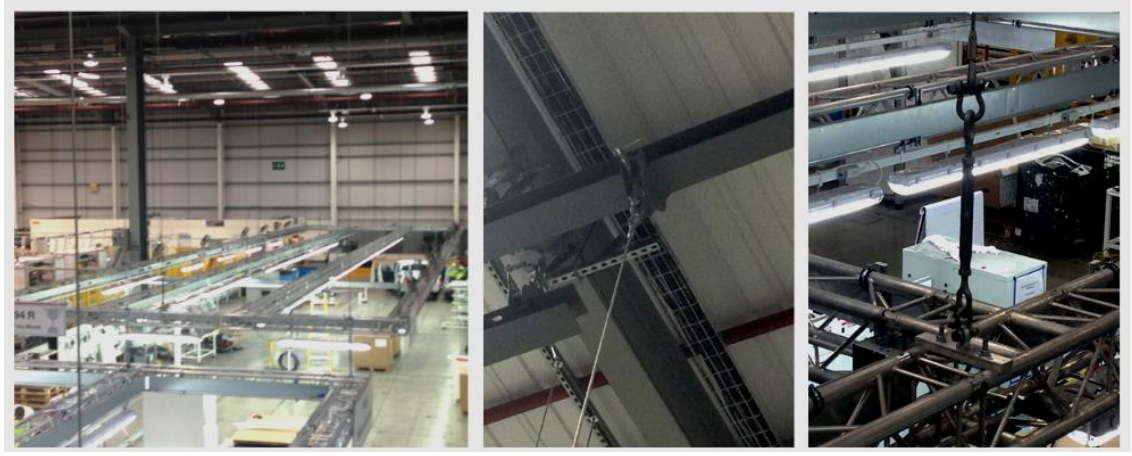

Fig. 1. Load bearing cables.

\footnotetext{
${ }^{*}$ Corresponding author: daniel.papan@ fstav.uniza.sk
} 
The cables are approximately $6.5 \mathrm{~m}$ long. They are made of six strand wire rope. Steel ropes are vertically anchored to the building structure of the production hall (horizontal load-bearing elements) and fixed to the rope itself (Fig. 1). For the purpose of the case study was the dynamic diagnostic chosen. In order to determine the tensile forces $T_{i}[\mathrm{kN}]$ in the individual hinges, it was necessary to define the basic relationships between dynamic parameters and tensile forces.

\section{Theoretical approach}

The drawn rope is considered as one-dimensional element. It can propagate waves that are by a one-dimensional differential equation expressed (1).

$$
\frac{\partial^{2} u}{\partial t^{2}}=v^{2} \frac{\partial^{2} u}{\partial x^{2}}
$$

The function $\mathrm{u}(x, t)$ is the instantaneous deflection of the rope element. It depends on the position $x$ on the rope and from time $t$. The parameter $v$ is the waves propagate speed along the rope. It depends on the thickness of the rope. It can be more precisely express as weight per unit of its length (length weight $s$ ) and from the tension force $F_{o}$. When we generate a harmonic (sinusoidal) propagation across a rope, the function $u_{1}(x, t)$ can be expressed by the equation (2).

$$
u_{1}(x, t)=A_{1} \sin \left[\omega_{1}\left(t-\frac{x}{v}\right)\right]=A_{1} \sin \left[2 \pi f_{1}\left(t-\frac{x}{v}\right)\right]
$$

The sine argument has a positive sign, which takes into account the opposite direction of the wave along the $x$ axis [2]. The reflected wave has always the same frequency as the incident wave. By reflection the deflection change to negative sign. The opposite waves with the same frequencies propagate through the cable and the final deflection corresponds the sum of the deflections of individual waves (Fig. 2).
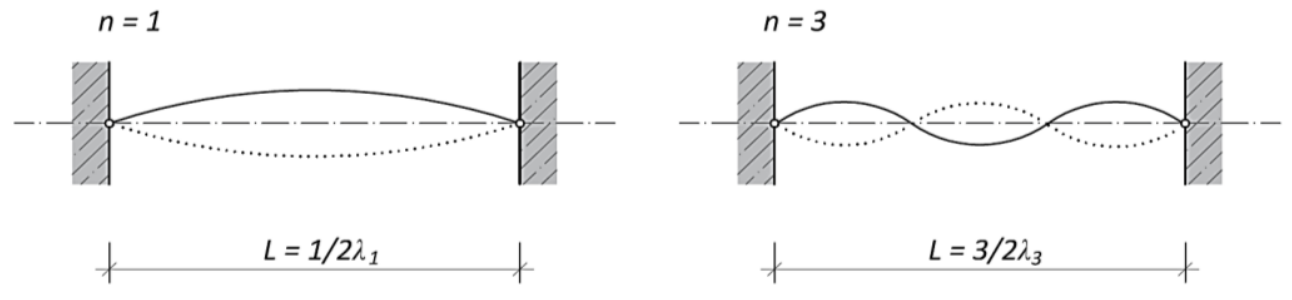

$n=2$

$$
n=4
$$
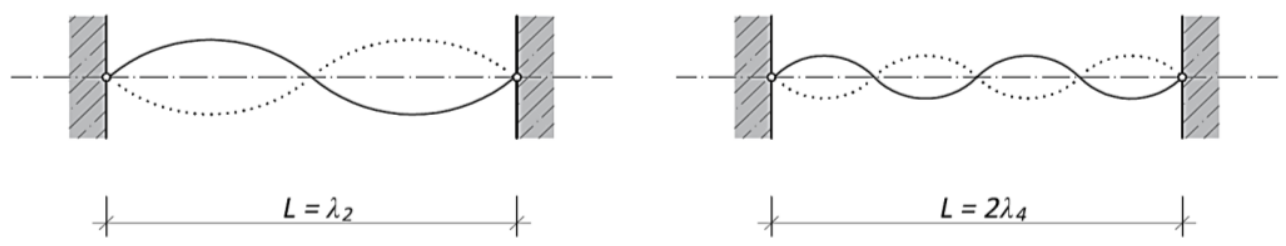

Fig. 2. Theoretical Basic modes of the cable.

At the cable support point $(x=0)$ is zero deflection. This means that the supported joint of the cable cannot vibrate. The situation is different if the other joint of the cable is fixed. Only waves of certain frequencies can propagate along the cable. The relationship between 
the frequency $f$, the wavelength $\lambda$ and the velocity $v$ of the harmonic wave can be expressed by the equation (3)

$$
v=f \cdot \lambda
$$

The wave propagation speed $(v)$ depends on the cable thickness $(s)$ and the tension force $\left(T_{\mathrm{o}}\right) \cdot(4)$

$$
v=\sqrt{\frac{T_{0}}{s}}
$$

The basic frequency of the cable vibration is called $f_{1}$ and the others are higher harmonic frequencies. The cable frequencies fixed at both ends may be only multiples of the base frequency. The basic wavelength $\lambda_{1}$ of the harmonic waves is equal to twice the length $L$ of the cable and all other wavelengths are fractions thereof (5).

$$
f_{1}=\frac{v}{2 L} ; \quad f_{n}=n \cdot f_{1}=n \cdot \frac{v}{2 L}=n \cdot \frac{f_{n} \cdot \lambda_{n}}{2 L} \quad \Rightarrow \quad \lambda_{n}=\frac{2 L}{n}=\frac{\lambda_{1}}{n}
$$

If we institute the equation (4) into the equation (5), we get the final evaluation of tensile forces (6).

$$
f_{1}=\frac{1}{2 L} \sqrt{\frac{T_{0}}{s}} ; \quad f_{\mathrm{i}}=\frac{1}{2 L} \sqrt{\frac{T_{\mathrm{i}}}{s}}
$$

\section{Dynamic diagnostic of cable system}

In order to determine the tensile forces in the suspension cables, it is necessary to determine the natural frequencies of the cable vibration. This is possible by transforming the time records of the vibrations of the individual cables into the frequency domain [3].

For this purpose is Fourier Transform (FT) used. FT is an expression of a timedependent signal using harmonic signals - sine and cosine functions. Discrete Fourier Transform (DFT) is the most used transformation by which we can calculate the frequency spectrum of a discrete signal. This spectrum is symmetric and complex conjugate around its central point. In practice, the most effective algorithm uses Fast Fourier Transform (FFT). With FFT we get the same results as with DFT, but much faster. For numerical solution of the frequency spectrum of a periodic continuous signal from Fourier series we use the basic equation (7).

$$
Y_{(k)}=\frac{1}{\tau} \cdot \int_{-\tau / 2}^{\tau / 2} x_{(t)} \cdot e^{-j \omega k t} d t
$$

Euler's equation can be expressed by the goniometric functions of Sinus and Cosine (8).

$$
y_{(k)}=\frac{1}{N} \cdot \sum_{n=0}^{N-1} x_{(n)} \cdot\left(\cos \left(2 \pi k \frac{n}{N}\right)+i \cdot \sin \left(2 \pi k \frac{n}{N}\right)\right)
$$

\subsection{Experimental measurement of dynamic vibration response of cable system}

For experimental measurement was used original designed line by Brüel \& Kjær with system PULSE [4]. The measurement line contents accelerometer, coaxial cable, modul and PC. Connection plan is on the figure (Fig.3). 


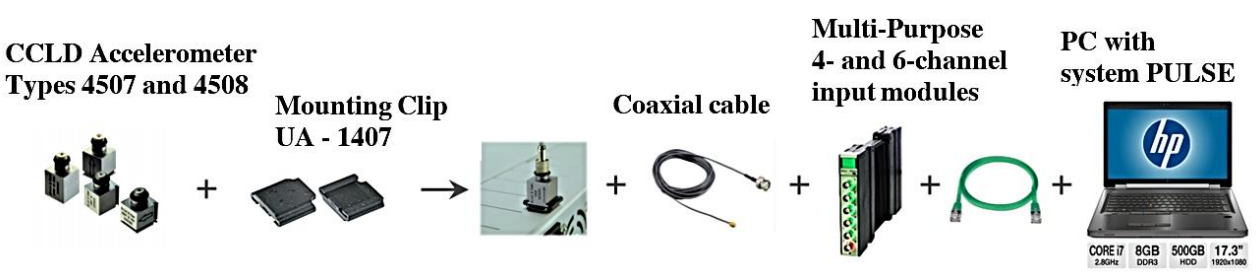

Fig. 3. Measurement line.

Used accelerometer (type BK 4508) was sequentially replaced on individual cables. It was fixed on magnetic pad and then placed to each suspension cable. The marks of individual cables and photo documentation is shown in the figure (Fig.4).
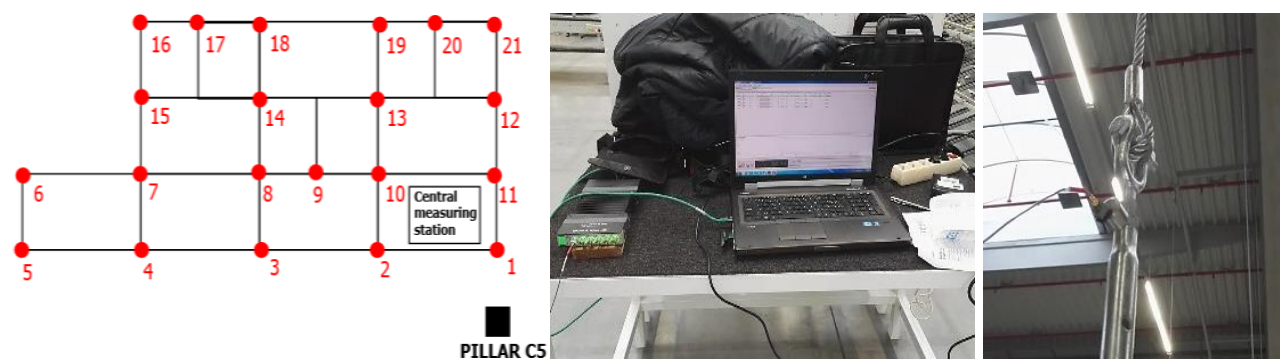

Fig. 4. Layout scheme and photo documentation.

During the measurement were at each point (from 1 to 21 ) recorded vibration accelerations. The sampling frequency was set to the $200 \mathrm{~Hz}$. The sensitivity of the measuring technique is significantly higher than the exciting impulse. For this reason was used only very soft excitation to vibrate the cable. A part of the system PULSE - Data Recorder - was used to register the vibration acceleration. In the figure (Fig.5 and 6) are shown specimen of vibration acceleration time records and frequency spectrum for some of the cables [5].
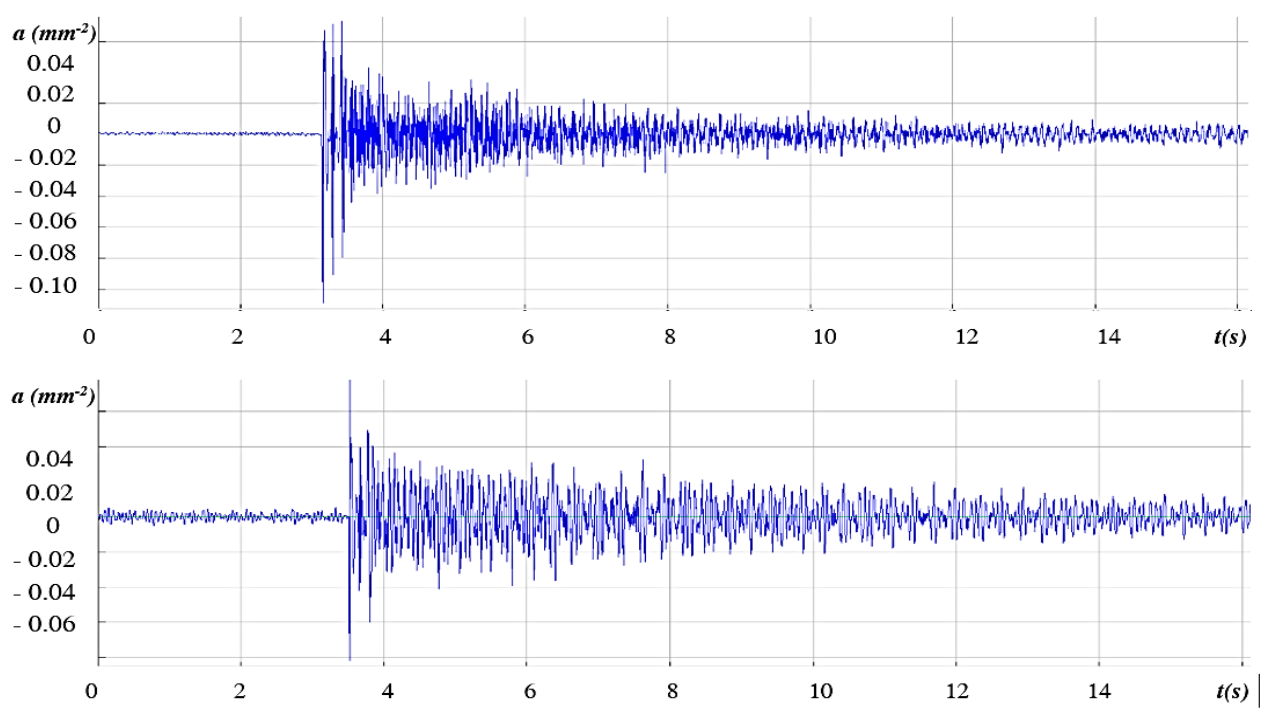

Fig. 5. Vibration acceleration time records of cables number 9 and 15. 

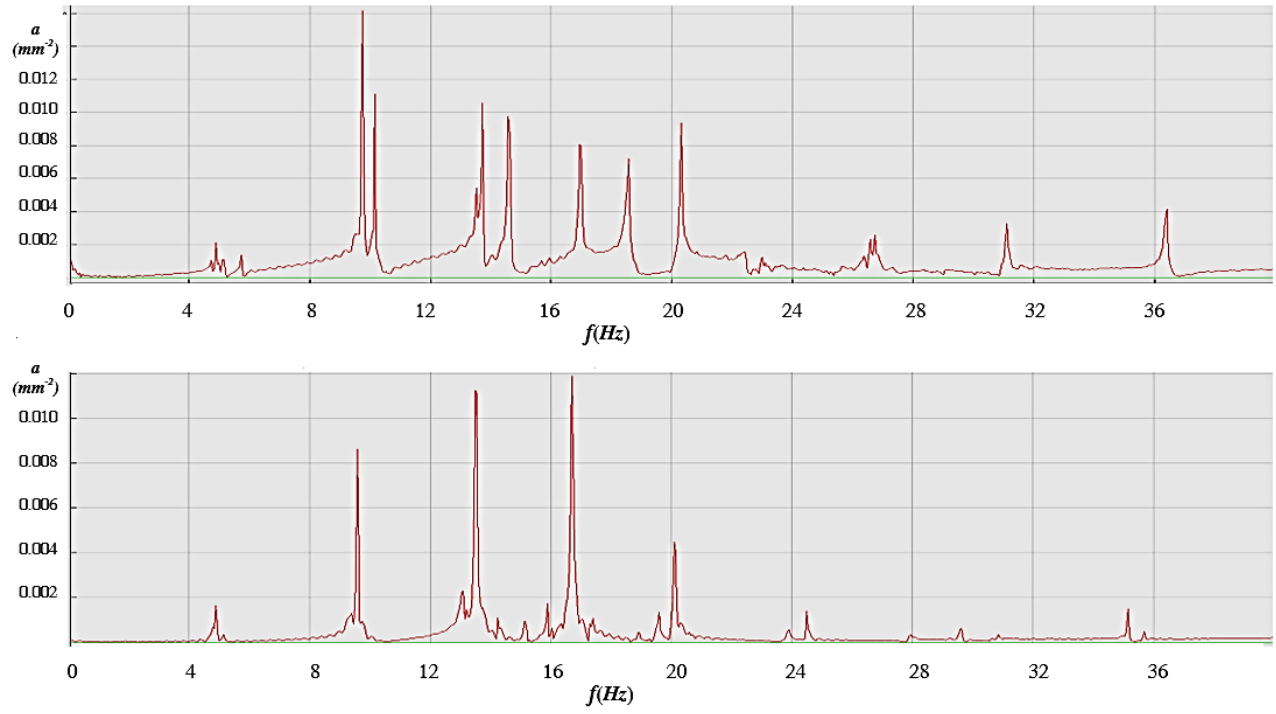

Fig. 6. Frequency spectrum of cables number 9 and 15.

The above equation (7) was used for finally data evaluation. The employed parameters were $g=0,3 \mathrm{~kg} / \mathrm{m} ; L=6,5 \mathrm{~m}$. A summary of the results is given in Table 1 .

Table 1. Data evaluation: frequencies $-f_{\mathrm{i}}[\mathrm{Hz}]$, tension forces $-T_{\mathrm{i}}[\mathrm{kN}]$

\begin{tabular}{|c|c|c|c|c|c|c|c|c|c|c|c|c|}
\hline $\mathbf{C}$ & $f_{1}$ & $T\left(f_{1}\right)$ & $f_{2}$ & $T\left(f_{2}\right)$ & $f_{3}$ & $T\left(f_{3}\right)$ & $f_{4}$ & $T\left(f_{4}\right)$ & $f_{5}$ & $T\left(f_{5}\right)$ & $\max T_{\mathrm{i}}$ & $\min T_{\mathrm{i}}$ \\
\hline $\begin{array}{ll}1 \\
\end{array}$ & 5.5 & 1.53 & 10 & 1.27 & 13.4 & 1.01 & 17.2 & 0.94 & 21.3 & 0.92 & 1.53 & 0.92 \\
\hline 2 & 4.9 & 1.20 & 9.9 & 1.23 & 13.6 & 1.04 & 16.9 & 0.90 & 20.3 & 0.84 & 1.23 & 0.84 \\
\hline 3 & 5.9 & 1.78 & 11.5 & 1.68 & 16.3 & 1.49 & 20.6 & 1.35 & 24.0 & 1.17 & 1.78 & 1.17 \\
\hline 4 & 6.1 & 1.90 & 12.1 & 1.84 & 16.7 & 1.57 & 20.6 & 1.34 & 25.1 & 1.28 & 1.90 & 1.28 \\
\hline 5 & 4.8 & 1.17 & 9.4 & 1.11 & 13.4 & 1.01 & 16.8 & 0.89 & 19.5 & 0.77 & 1.17 & 0.77 \\
\hline 6 & 4.4 & 0.97 & 8.4 & 0.90 & 11.8 & 0.79 & 14.9 & 0.70 & 18.1 & 0.66 & 0.97 & 0.66 \\
\hline 7 & 6.4 & 2.06 & 11.9 & 1.80 & 16.0 & 1.44 & 19.3 & 1.18 & 23.8 & 1.15 & 2.06 & 1.15 \\
\hline 8 & 5.8 & 1.71 & 11.6 & 1.71 & 17.0 & 1.63 & 21.6 & 1.48 & 26.5 & 1.42 & 1.71 & 1.42 \\
\hline 9 & 7.5 & 2.84 & 14.6 & 2.69 & 16.4 & 1.51 & 18.6 & 1.09 & 26.7 & 1.45 & 2.84 & 1.09 \\
\hline 10 & 7.6 & 2.90 & 10.4 & 1.38 & 13.3 & 1.00 & 18.9 & 1.13 & 23.4 & 1.11 & 2.90 & 1.00 \\
\hline 11 & 6.9 & 2.44 & 9.5 & 1.13 & 12.1 & 0.82 & 14.9 & 0.71 & 18.5 & 0.70 & 2.44 & 0.70 \\
\hline 12 & 4.0 & 0.83 & 7.8 & 0.76 & 10.9 & 0.67 & 13.8 & 0.60 & 17.1 & 0.59 & 0.83 & 0.59 \\
\hline 13 & 4.0 & 0.83 & 7.9 & 0.79 & 11.2 & 0.70 & 14.2 & 0.64 & 17.2 & 0.60 & 0.83 & 0.60 \\
\hline 14 & 4.7 & 1.13 & 9.1 & 1.05 & 12.9 & 0.94 & 16.1 & 0.82 & 19.7 & 0.78 & 1.13 & 0.78 \\
\hline 15 & 4.9 & 1.22 & 9.6 & 1.17 & 13.5 & 1.03 & 16.7 & 0.88 & 20.1 & 0.82 & 1.22 & 0.82 \\
\hline 16 & 2.9 & 0.41 & 5.7 & 0.41 & 8.3 & 0.39 & 9.6 & 0.29 & 11.6 & 0.27 & 0.41 & 0.27 \\
\hline 17 & 5.6 & 1.35 & 10.1 & 1.30 & 14.6 & 1.20 & 18.5 & 1.08 & 22.7 & 1.04 & 1.35 & 1.04 \\
\hline 18 & 3.3 & 0.57 & 6.5 & 0.53 & 9.2 & 0.47 & 11.5 & 0.42 & 14.2 & 0.41 & 0.57 & 0.41 \\
\hline 19 & 3.3 & 0.56 & 4.6 & 0.27 & 5.7 & 0.18 & 7.0 & 0.16 & 8.8 & 0.16 & 0.56 & 0.16 \\
\hline 20 & 5.8 & 1.71 & 10.5 & 1.40 & 15.3 & 1.31 & 18.2 & 1.05 & 22.2 & 1.00 & 1.71 & 1.00 \\
\hline 21 & 3.3 & 0.57 & 6.6 & 0.54 & 9.9 & 0.55 & 12.6 & 0.50 & 17.0 & 0.59 & 0.59 & 0.50 \\
\hline
\end{tabular}




\section{Conclusions}

This paper shows how the simple theory of tensioned cable vibration can be applied to determination of the tension force. This specific practical request was solved theoreticexperimental way. For this purpose was used excellent and sensitive experimental equipment. Also the specific dynamic excitation was performed. The random paraseismic waves due to forklifts and the other technological machines in the industrial process generated the cable excitation [6]. This excitation was selected because it contains sufficient frequency band for the natural vibration of expected tension forces in cables. The results of this study can help designers with assessment of such complicated structure. This approach is one of the best solution when the manufacturing process cannot be stopped and for structural investigation. According Slovak standards the maximum tensile force in cable 11 is $2.9 \mathrm{kN}$ was save for the next lifetime of the structure.

This paper was created with the support of Faculty of Civil Engineering, Department of Structural Mechanics and Applied Mathematics, University of Žilina and laboratories technical equipment of the institution.

\section{References}

1. B. Valent, Static design of processing part H1 - Jaguar Land Rover, (2016)

2. T. C. A. Molteno, N. B. Tufillaro, An experimental investigation into the dynamics of a string, AJP, 72 (2004).

3. N. B. Tufillaro, Nonlinear and chaotic string vibrations, AJP, 57 (1989).

4. Electronic document: https://www.bksv.com/media/doc/bu0228.pdf (2019)

5. D. Papan, Z. Papánová, Spectral Analysis of the Structures due to Traffic, (XXIII R-SP Seminar, 91, 2014)

6. D. Papan, Z. Papánová, Numerical models for the elastic halfspace dynamic response due to road traffic load, MATEC Web of Conferences, 251 (2018) 